\title{
Preoperative intensity-modulated chemoradiotherapy with simultaneous integrated boost in rectal cancer: five-year follow-up results of a phase II study
}

\author{
Jasna But-Hadzic ${ }^{1,2}$, Anja Meden Boltezar ${ }^{1}$, Tina Skerl'1 , Vesna Zadnik²,3, Vaneja Velenik ${ }^{1,2}$ \\ ${ }^{1}$ Institute of Oncology Ljubljana, Division of Radiotherapy, Ljubljana, Slovenia \\ 2 Faculty of Medicine, University of Ljubljana, Ljubljana, Slovenia \\ ${ }^{3}$ Institute of Oncology Ljubljana, Epidemiology and Cancer Registry, Ljubljana, Slovenia
}

Radiol Oncol 2021; 55(4): 439-448.

Received 17 February 2021

Accepted 26 April 2021

Correspondence to: Assist. Prof. Jasna But-Hadžić, M.D., Ph.D., Institute of Oncology Ljubljana, Zaloška 2, 1000 Ljubljana, Slovenia. E-mail: jbut@onko-i.si

Disclosure: No potential conflicts of interest were disclosed.

This is an open access article under the CC BY-NC-ND license (http://creativecommons.org/licenses/by-nc-nd/4.0/).

Background. We conducted a phase II study to investigate the feasibility and safety of preoperative radiochemotherapy experimental fractionation, using intensity-modulated radiation therapy with simultaneous integrated boost (IMRT SIB) to shorten the overall treatment time without dose escalation in intermediate/locally advanced rectal cancer with the aim to improving treatment outcome.

Patients and methods. A total of 51 patients with operable stage II-III rectal carcinoma were included between January 2014 and January 2015. Fifty patients completed preoperative IMRT treatment with an elective dose of 41.8 Gy and simultaneously delivered 46.2 Gy to T2/T3 and 48.4 Gy to T4 tumour in 22 fractions, with concomitant capecitabine ( $825 \mathrm{mg} / \mathrm{m}^{2} / 12 \mathrm{~h}$, including at weekends). Median follow-up was 70 months (range 11-80 m).

Results. Forty-seven patients completed treatment per protocol. Acute toxicity occurred in 2 (4\%) patients. RO resection was achieved in all but 1 and pathologic complete response (PCR) in 12 (25.5\%) patients who had 5-year overall survival (OS), disease-free survival (DFS) and local control (LC) of $91.7 \%, 100 \%$ and 100\%, respectively. The intentionto-treat analysis showed that the type of surgery significantly moderated OS and DFS, while total downstaging and pN were predictive for DFS only. For treatment per protocol 5-year OS, DFS and LC were $80.9 \% 195 \%$ confidence interval [Cl] 69.7-92.1), 77.1\% (95\% Cl 65.1-89.1) and 95.2\% (95\% Cl 88.7-100), respectively. The proportion of patients with severe late (CTCAE G $\geq 3$ ) gastrointestinal, urinary and sexual toxicity was $15 \%, 2 \%$ and $8 \%$ respectively, with one reported secondary carcinoma.

Conclusions. Preoperative IMRT-SIB without dose escalation was well tolerated, with a low acute toxicity profile, we achieved a high rate of $\mathrm{PCR}$ and showed encouraging 5-year OS, DFS and LC.

Key words: rectal cancer; IMRT; simultaneous integrated boost; preoperative radiochemotherapy; acute toxicity, pathologic complete response; overall survival; disease-free survival; local control; late toxicity; quality of life

\section{Introduction}

In recent years, many different treatment strategies have been tested to improve outcomes for patients with locally advanced rectal cancer, with toxicity being the main obstacle for intensification of the standard treatment. ${ }^{1-4}$ Changing the preoperative radiotherapy (RT) technique from 3D conformal (3D CRT) to intensity-modulated radiotherapy (IMRT) allowed better sparing of normal tissue in dosimetric analyses ${ }^{5-8}$ and was used in several phase II studies to achieve dose escalation with si- 
multaneous integrated boost (SIB) with or without another drug in addition to standard concomitant capecitabine. $^{9-14}$ The published reports showed encouraging results for pathologic complete response (pCR) and local control (LC) ${ }^{9,11}$, but with no impact on gastrointestinal toxicity with the addition of oxaliplatin ${ }^{15}$ and important late toxicity with dose escalation. ${ }^{10}$

Due to the promising impact on clinical outcome, but, conflicting toxicity results of treatment intensification with IMRT-SIB dose escalation in preoperative locally advanced rectal cancer (LARC) treatment, we conducted a prospective phase II study, where we kept the biologically effective dose (BED) of experimental IMRT SIB fractionation similar to the standard 3D CRT protocol of $45 \mathrm{~Gy}$ elective dose and boost of $4.5 \mathrm{~Gy}$ to $\mathrm{T} 3$ and 9 Gy to T4 tumour.

Our previously published results have shown that radiochemotherapy with IMRT-SIB without dose escalation, concomitantly with capecitabine, achieved a high rate of pCR $(25.5 \%)$ and downstaging rate, with favourable acute toxicity profile and excellent compliance. ${ }^{16}$ In this paper, we report LC, disease-free survival (DFS), overall survival (OS), late toxicity and quality of life (QoL) after median follow-up of 70 months.

\section{Patients and methods}

\section{Study design and inclusion criteria}

Details about the trial (the eligibility criteria, treatment details and trial design) are available elsewhere. ${ }^{16,17}$ In short, to enter the study patients had to present with operable, histologically proven, intermediate/locally advanced (cT $\geq 3$ and/or $\mathrm{cN} \geq 1$ on MRI), non-metastatic (M0 confirmed on CT thorax and abdomen) rectal adenocarcinoma, located up to $15 \mathrm{~cm}$ from the anal verge with no contraindications for systemic therapy. Written consent was signed before entering the trial, which was approved by the National Medical Ethics Committee of the Republic of Slovenia (No. 41/12/13) and complied with the Declaration of Helsinki. The study was registered in the ClinicalTrials.gov database (NCT02268006).

\section{Treatment protocol}

The target volumes and dose prescription were described in detail. ${ }^{16}$ Visible primary tumour was contoured as the gross tumour volume (GTV) and was extended with a $1 \mathrm{~cm}$ margin to represent a boost volume (clinical target volume 2 - CTV2). Clinical target volume 1 (CTV1) encompassed CTV2, mesorectum, and regional lymph nodes. The nodes along the arteria illiaca externa were included in case of substantial genitourinary structure infiltration, and the ischiorectal fossa and anal canal if the musculus levator ani or anal canal were involved. CTV 1 was extended anteriorly due to bowel movement as internal target volume (ITV). ITV $+1 \mathrm{~cm}(7 \mathrm{~mm}$ posterior/lateral) represented the planning target volume (PTV). PTV 1 received 41.8 Gy in 22 fractions and SIB was prescribed to tumour (PTV 2) concomitantly to doses of $46.2 \mathrm{~Gy}$ and 48.4 Gy to $\mathrm{T} \leq 3$ and T4 tumours in 22 fractions, respectively, 5 times per week (Monday to Friday). Concomitant capecitabine was prescribed from the first to the last day of the radiation treatment (including at weekends) at a daily dose of $825 \mathrm{mg} / \mathrm{m}^{2} / 12 \mathrm{~h}$. The treatment was delivered on Clinac 2100 CDI (Varian, Palo Alto, USA) using the dynamic multileaf collimator technique with $6 \mathrm{MV}$ photons and a daily position verification (ExacTrac X-ray 6D system, BrainLAB AG, Feldkirchen, Germany).

After total mesorectal excision (TME), that was scheduled 6-8 weeks after preoperative treatment, six cycles of adjuvant chemotherapy with capecitabine were offered to patients with residual tumour on pathologic examination. Pathologic stage and tumour regression grade (TRG) were recorded according to the American Joint Committee on Cancer (AJCC) 7 th edition ${ }^{18}$ and criteria by Dworak et al. ${ }^{19}$, respectively.

All patients were followed up with clinical and serum CEA evaluation every 3 months for two years, and later on a bi-annual basis with abdominal ultrasound every 6 months and a chest radiograph annually.

\section{Statistics}

This prospective phase II study in patients with intermediate/locally advanced rectal cancer was designed to evaluate the pathologic complete response after experimental preoperative treatment as a primary endpoint. The key secondary endpoints were to evaluate the acute toxicity of preoperative treatment, tumour response, local control (LC), disease-free survival (DFS) and overall survival (OS). In this report, we focus on survival, late toxicity and quality of life (QoL) after a 5-year follow-up.

A statistical analysis was performed with the Statistical Package for Social Sciences, v. 25.0 (SPSS 
Inc., Chicago, IL, USA). Descriptive statistics were used to present frequencies. Survival was calculated with the Kaplan-Meier method and the influence of possible prognostic factors verified by means of the log-rank test. Time intervals were defined from the end of treatment (operation or radiotherapy completion for non-operated patients) until the last follow-up or death for OS and additionally until local or distant recurrence for DFS. For the intentionto-treat analysis (all patients), LC and DFS were counted as $0 \mathrm{~m}$ for non-operated patients and DFS as $0 \mathrm{~m}$ for M1 patients. Patients surgically treated after chemoradiotherapy completion $(\mathrm{N}=47)$ entered treatment per protocol analysis.

Late adverse events data were available in the medical records for all patients and telephone interviews were additionally performed in November 2020, discussing patients' late adverse effects and quality of life, following the Common Terminology Criteria for Adverse Events (CTCAE) v 5.0. ${ }^{20}$

QoL was recorded with the EORTC cancer-specific core questionnaire QLQ-C $30^{21}$ and colorectalspecific questionnaire QLQ-C2922, that were collected before treatment (T0), and 1 year (T1) and 5 years (T5) after treatment. Data from all questionnaires were available for 31 patients for which the recorded answers were transformed into dimensions in the range $1-100 .{ }^{23}$ Higher scores represented a higher level of functioning (for functional scales and single items) and lower scores displayed a lower symptom level (for symptom scales and single items). Statistical significance for QLQ scores changes over time was verified by comparing means with the Wilcoxon signed-rank test and with the t-test for EORTC reference value comparison. A p-value < 0.05 was considered statistically significant.

\section{Results}

Between January 2014 and January 2015, 51 (N = 51) patients were included. The patients' characteristics are described in detail elsewhere ${ }^{16}$, but brief$1 y$ - median age was 66 years (range: $33-81$ years) and nearly half of the tumours were located in the lower third of the rectum. The tumour invaded the mesorectal fascia in 20 patients and 15 patients had suspicious extramesorectal lymph nodes on MRI. Clinical stages were: T2N1M0 $(n=1)$, T3N0M0 (n $=6)$, T3N1M0 ( $=15)$, T3N2M0 $(n=22)$, T4N1M0 $(\mathrm{n}=4), \mathrm{T} 4 \mathrm{~N} 2 \mathrm{M} 0(\mathrm{n}=2)$, and T3N1M1 ${ }^{18}$, with small lung lesion prior to inclusion revealed as lung metastasis on control CT after the treatment in the last patient.
Preoperative radiochemotherapy was completed by 50 patients and 1 received preoperative short-course radiotherapy due to ischaemic stroke.

Altogether, 48 patients underwent surgery ( 47 treated per protocol). In 3 patients, surgery was omitted due to patient refusal, synchronous pancreatic cancer and rectal varices haemorrhage. Low anterior resection (LAR) was performed in 40 patients, abdominoperineal resection (APE) in 7 , and pelvic exenteration in 1 . One patient had a positive circumferential margin. Extramesorectal nodes exploration was based upon surgeon discretion and nodes were removed in 4 patients.

The total downstaging rate was $87 \%$ (41/47 patients), with a decrease in $\mathrm{T}$ and $\mathrm{N}$ stage observed in 32 and 39 patients, respectively. Pathologic complete response was achieved in 12 patients.

In median follow-up of 70 months (range 11-80 m) we recorded 13 deaths, 7 due to rectal cancer. Among the 6 remaining patients, 3 died of cardiovascular disease and one each of pancreatic cancer, alcohol hepatic cirrhosis and grade 5 (G5) ileus. One isolated local relapse and 1 with synchronous distant metastasis occurred 41 and 42 months after LAR and APE, respectively. Time to distant relapses was $0 \mathrm{~m}$ and $18 \mathrm{~m}$ (lung), 6 and $11 \mathrm{~m}$ (liver), $41 \mathrm{~m}$ (adrenal gland) and $42 \mathrm{~m}$ (abdominal lymph nodes). At the latest date of follow-up on 30.1.2021, there were 37 patients alive without disease and one patient on systemic treatment for disseminated rectal cancer.

\section{Survival}

We performed an intention-to-treat analysis for all 51 patients and for 47 patients that were treated according to protocol (Table 1). For the entire cohort, cumulative 5-year OS, 5-year DFS and 5-year LC were $76.5 \%$ (95\% CI 64.9-88.1), 72.4\% (95\% CI 60.484.6), and $89.7 \%$ (95\% CI 81.1-98.3), respectively. In the treatment per protocol group 5-year OS, 5-year DFS and 5-year LC were 80.9\% (95\% CI 69.7-92.1), 77.1\% (95\% CI 65.1-89.1) and 95.2\% (95\% CI 88.7$100)$, respectively. Five-year colostomy-free survival was $76 \%(29 / 38)$.

The potential influence of prognostic factors on survival was determined by means of the log-rank test (Table 2). There was no association between age at diagnosis, performance status, tumour grade, positive mesorectal fascia or suspicious extramesorectal lymph nodes, removal of ekstramesorectal lymph nodes, clinical stage $(\mathrm{cT}, \mathrm{cN})$, decrease in $\mathrm{T}$ and $\mathrm{N}$ stage or pathologic $\mathrm{T}$ stage on survival. We found no predictive value for $\mathrm{PCR}$, TRG prognostic 
TABLE 1. Number of events after median follow-up of 70 months $(11-80 \mathrm{~m})$ and 5-year survival

\begin{tabular}{lcc}
\hline & $\begin{array}{c}\text { Intention to treat } \\
(\mathbf{N}=51)\end{array}$ & $\begin{array}{c}\text { Per protocol } \\
(\mathbf{N}=\mathbf{4 7})\end{array}$ \\
\hline 5-year OS* & $76.5 \%$ & $80.9 \%$ \\
5-year DFS & $72.5 \%$ & $76.5 \%$ \\
5-year LC & $90.2 \%$ & $95.7 \%$ \\
\hline OS status & Number of events (\%) & Number of events (\%) \\
\hline Alive & $38(74.5)$ & \\
\hline Dead & $13(25.5)$ & $37(78.7)^{*}$ \\
\hline DFS status & $37(72.5)$ & $10(21.3)$ \\
\hline Alive without disease & $14(27.5)$ & $36(76.5)$ \\
Local/distant relapse/death & & $11(23.5)$ \\
\hline LC status & $46(90.2)$ & $45(95.7)$ \\
\hline Local relapse - & $5(9.8)$ & $2(4.3)$ \\
\hline Local relapse + & & \\
\hline
\end{tabular}

* = Numbers differ from OS status due to one noncancer death > 5-year after surgery; DFS = disease-free survival; $L C=$ local control; OS = overall survival; for non-operated patients and patient with $\mathrm{M}+$ disease local or distant recurrence was calculated as 0 months. group or NAR prognostic group as survival surrogates. Significantly better DFS was found in patients where total downstaging was achieved and in patients with pathologically negative lymph nodes (Figure 1). There was significantly better OS and DFS with LAR compared with APE or pelvic exenteration ( $\mathrm{p}=0.000$ and 0.013 , respectively). Gender was a predictive prognostic factor for OS and treatment per protocol was associated with better OS and DFS.

We recorded no local or distant relapses in the group of patients with $\mathrm{pCR}$, with one death due to G5 adverse event, leading to 91.7\% 5-year OS and $100 \%$ 5-year DFS and 5-year LC for this group of patients.

\section{Late toxicity}

Late toxicity data are available for all patients and are listed in Table 3. Patients reported mean 5 late adverse events (range 0-19) at last follow up. Major adverse events (CTCAE version $5.0 \mathrm{G} \geq 3$ ) $^{20}$
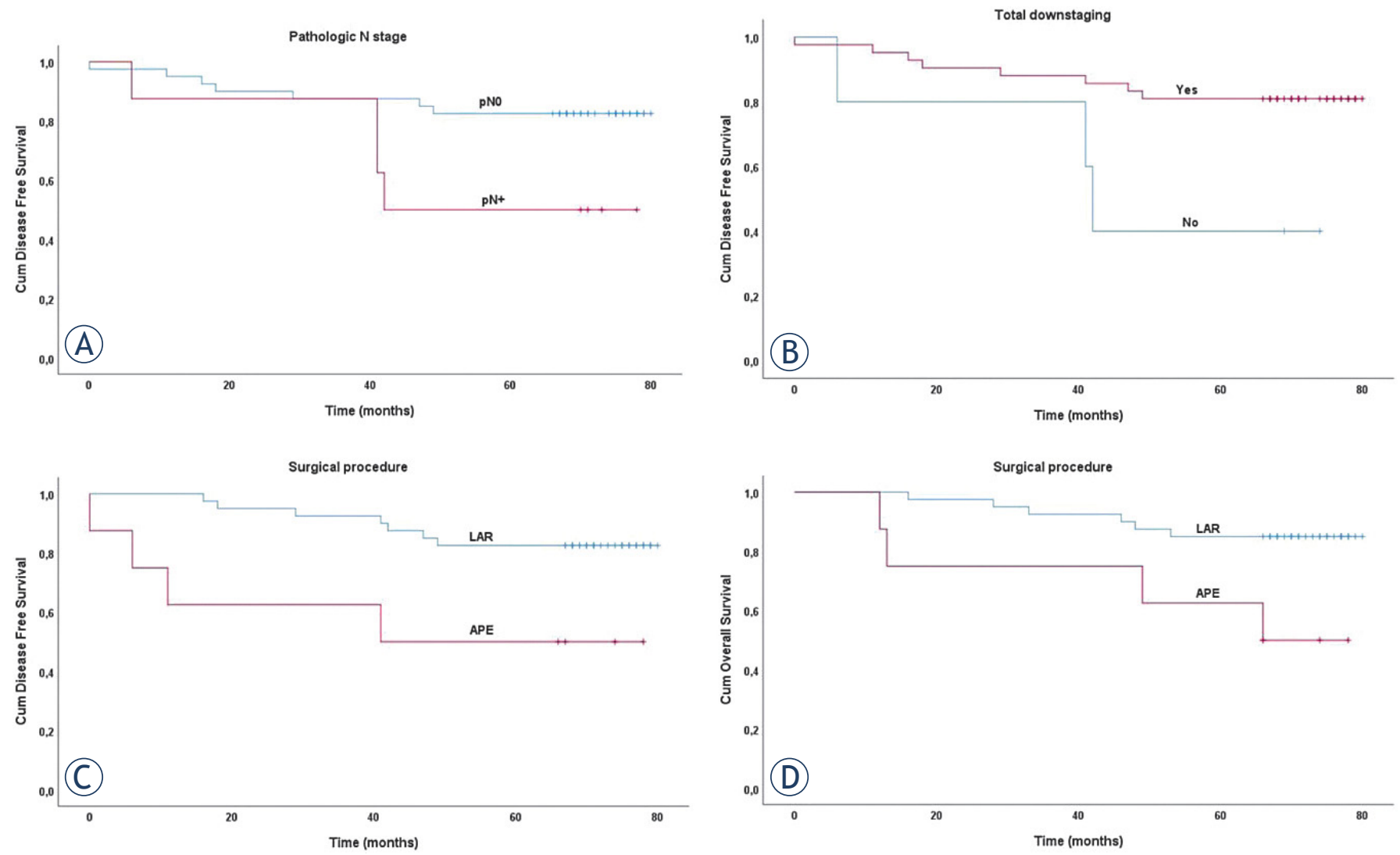

FIGURE 1. Prognostic significance of (A) pathologic nodal stage ( $\mathrm{pN}$ ) and (B) total downstaging on 5-year disease-free survival, (C) prognostic significance of surgery procedure on 5-year disease-free survival and (D) overall survival in rectal cancer after preoperative radiochemotherapy and surgery.

$\mathrm{APE}=$ abdominoperineal excision; $\mathrm{LAR}=$ low anterior resection 
occurred in 12 patients (23\%), of which 7 patients had one, 3 patients two and 1 patient had three G3 toxicities. Following protective stoma closure, 1 patient died due to G5 ileus complication 29 months after LAR. Gastrointestinal toxicity (GI) $\geq$ 3 was recorded in 7 (15\%) and genitourinary (GU) in $5(10 \%)$ patients. Two men have erectile dysfunction and two women are reporting problems due to dyspareunia, vaginal dryness and vaginal stricture. Due to complete faecal incontinence, permanent stoma was required in two patients, 10 and 36 months after LAR. Urgent surgical intervention was required for anastomotic dehiscence and hernia incarceration in one case where the patient later developed enterocutaneous fistula. In the remaining two patients with anastomotic dehiscence, protective stoma closure was omitted in one patient and permanent stoma was placed 23 months after LAR in the other patient. Permanent stoma placement was also required due to rectoprostatic fistula in one patient $36 \mathrm{~m}$ after LAR. Altogether, 6 patients with sphincter-preserving surgery had stoma closure omitted or later placed as permanent due to late toxicity (faecal incontinence, anastomotic dehiscence and fistula). The last recorded serious adverse event possibly related to treatment was recorded after $60 \mathrm{~m}$ of follow up in a patient with bladder carcinoma (Figure 2).

\section{Quality of life evaluation (QoL)}

Of 38 eligible patients, $31(81.6 \%)$ completed the EORTC QLQ-C30 and QLQ-C29 questionnaires before treatment (T0), 1 year (T1) and $\geq 5$ years (T5) after treatment at median age 75 years (range 37-86 years). The global QoL mean scores have not significantly changed over time (mean T0 vs. T5 was 57.0 vs. $60.8 ; \mathrm{p}=0.384$ ), but were significantly lower compared to the general Slovenian population $(p=0.035)$. Also, no significant differences in mean scores over time were observed for any of the core functional and physical scales (data shown in Supplement 1). Significant changes in CR29 scales occurred 1 year after treatment and remain significant $>5$-year post treatment. There was a significant drop in reported blood and mucus (mean T0 vs. $\mathrm{T} 1$ vs. $\mathrm{T} 5$ was 33.9 vs.7.5 vs.4.3; T0/T1 and T0/T5 $\mathrm{p}<0.000$ ) and anxiety score (mean T0 vs. T1 vs. T5 was 67.7 vs. 39.3 vs. $42.2 ; \mathrm{T} 0 / \mathrm{T} 1 \mathrm{p}=0.000$ and $\mathrm{T} 0 / \mathrm{T} 5$ $p=0.005)$, but higher scores were recorded for faecal incontinence/leakage, hair loss, and body image (T0/T1 $\mathrm{p}=0.027,0.046$, and 0.007 , respectively). There was no difference in mean scores for urinary incontinence between $\mathrm{T} 0 / \mathrm{T} 1$, but mean scores rose
TABLE 2. Influence of probable prognostic factors on OS and DFS

\begin{tabular}{|c|c|c|c|c|}
\hline \multirow[b]{2}{*}{ Prognostic factor } & \multicolumn{2}{|c|}{$\begin{array}{l}\text { Intention to treat } \\
\qquad(N=51)\end{array}$} & \multicolumn{2}{|c|}{$\begin{array}{l}\text { Per protocol } \\
(\mathrm{N}=47)\end{array}$} \\
\hline & OS & DFS & OS & DFS \\
\hline $\begin{array}{l}\text { Age at diagnosis } \\
\text { ( } \geq 65 \text { years vs. }<65 \text { years) }\end{array}$ & ns & ns & ns & ns \\
\hline $\begin{array}{l}\text { Gender } \\
\text { (male vs. female) }\end{array}$ & $p=0.044$ & ns & $p=0.064$ & ns \\
\hline PS WHO & ns & ns & ns & ns \\
\hline Tumour grade & ns & ns & ns & ns \\
\hline $\begin{array}{l}\text { Tumour location (upper/ } \\
\text { middle/lower rectal third) }\end{array}$ & ns & ns & ns & ns \\
\hline$M R I+$ & ns & ns & ns & ns \\
\hline $\begin{array}{l}\text { Extramesorectal lymph nodes } \\
\text { (positive/negative) }\end{array}$ & ns & ns & ns & ns \\
\hline $\begin{array}{l}\text { Time to treatment } \\
(\leq 7 w />7 w)\end{array}$ & $p=0.045$ & ns & ns & ns \\
\hline $\begin{array}{l}\text { Surgery procedure } \\
\text { (APE and pelvic } \\
\text { exenteration/LAR) }\end{array}$ & $p=0.000$ & $p=0.013$ & $p=0.020$ & $p=0.016$ \\
\hline CT stage ${ }^{a}$ & ns & ns & ns & ns \\
\hline cN stage ${ }^{a}$ & ns & ns & ns & ns \\
\hline Decrease in T stage & ns & ns & ns & ns \\
\hline Decrease in $\mathrm{N}$ stage & ns & ns & ns & ns \\
\hline Total downstaging & ns & $p=0.029$ & ns & $p=0.029$ \\
\hline pt stage (0-2 vs. $3-4)$ & ns & ns & ns & ns \\
\hline pN stage (0 vs. + ) & ns & $p=0.044$ & ns & $p=0.019$ \\
\hline $\begin{array}{l}\text { Ekstramesorectal lymp node } \\
\text { removal }\end{array}$ & ns & ns & ns & ns \\
\hline $\mathrm{PCR}$ & ns & ns & ns & ns \\
\hline TRG prognostic group & ns & ns & ns & ns \\
\hline NAR prognostic group & ns & ns & ns & ns \\
\hline $\begin{array}{l}\text { Adjuvant chemotherapyb } \\
\text { (5-6/ } 4 \text { cycles) }\end{array}$ & ns & ns & ns & ns \\
\hline Treatment per protocol & $p=0.006$ & $p=0.001$ & / & I \\
\hline
\end{tabular}

a according to AJCC, 7th edition ${ }^{18}$; ${ }^{b}$ calculated for 36 patients with indication for adjuvant chemotherapy; $\mathrm{APE}=$ abdominoperineal excision; $\mathrm{DFS}=$ disease free survival; $\mathrm{LAR}=$ low anterior resection; $\mathrm{MRI+}=$ positive mesorectal fascia; $\mathrm{N}=$ nodal; $\mathrm{NAR}=$ neoadjuvant rectal cancer score $^{32}$; $n s=$ not specific $(p>0.05)$. OS = overall survival; $\mathrm{pCR}=$ pathologic complete response; $\mathrm{PS}$ WHO = WHO performance status; $T=$ tumour; TRG = tumour regression grade $^{19}$

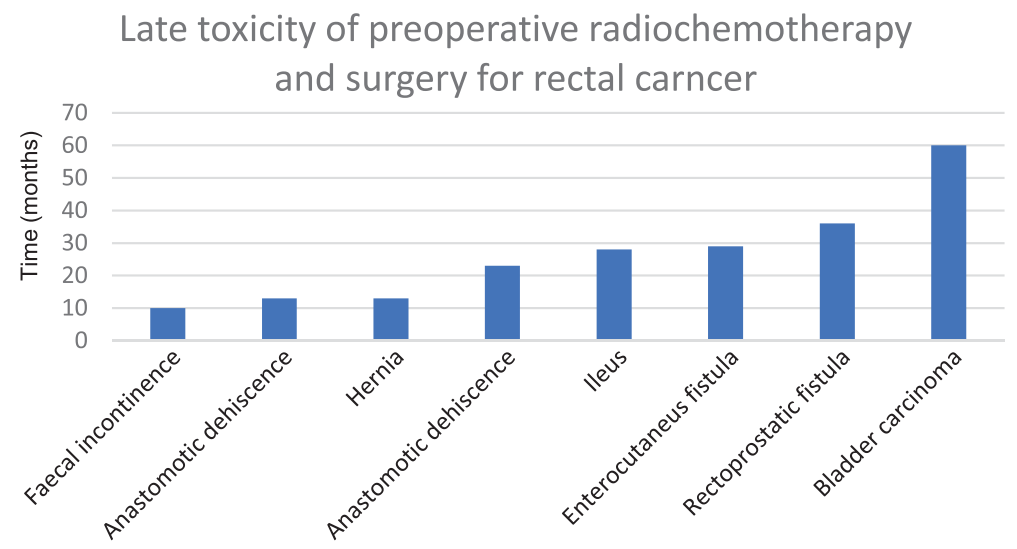

FIGURE 2. Time to occurrence of $G \geq 3$ adverse events. 
TABLE 3. Late toxicity after preoperative radiochemotherapy, surgery and adjuvant chemotherapy according to CTCAE version $5.0^{20}$

\begin{tabular}{|c|c|c|c|c|c|}
\hline & G1, n (\%) & G2, n (\%) & G3, n (\%) & G4, n (\%) & G5, n (\%) \\
\hline Anastomotic dehiscence & - & $1(2.1)$ & $3(6.3)$ & - & - \\
\hline Anastomotic stenosis & $4(10.0)$ & - & - & - & - \\
\hline lleus & - & - & - & - & $1(2.1)$ \\
\hline Hernia & $4(8.3)$ & $1(2.1)$ & $1(2.1)$ & - & - \\
\hline Abdominal or pelvic pain & $11(22.9)$ & $3(6.3)$ & - & - & - \\
\hline Anal stenosis & $5(10.4)$ & - & - & - & - \\
\hline Fistula & - & $1(2.1)$ & $2(4.2)$ & - & - \\
\hline Bloating & $21(43.8)$ & $3(6.3)$ & - & - & - \\
\hline Constipation & $10(20.8)$ & $4(8.3)$ & - & - & - \\
\hline Diarrhoea & $9(18.8)$ & $5(10.4)$ & - & - & - \\
\hline Faecal incontinence & $6(15.4)$ & $12(30.8)$ & $3(7.7)$ & - & - \\
\hline Faecal urgency* & $5(13.2)$ & $1(2.6)$ & - & - & - \\
\hline Flatulence & $25(52.1)$ & $6(12.5)$ & - & - & - \\
\hline Haemorrhoidal haemorrhage & $1(2.1)$ & - & - & - & - \\
\hline Haemorrhoids & $3(6.3)$ & - & - & - & - \\
\hline Proctitis & $1(2.1)$ & - & - & - & - \\
\hline Intestinal stoma leak & $2(8.3)$ & - & - & - & - \\
\hline Dysuria & $1(2.1)$ & - & - & - & - \\
\hline Urinary frequency & $13(27.1)$ & - & - & - & - \\
\hline Urinary incontinence & $9(18.8)$ & $4(8.3)$ & $1(2.1)$ & - & - \\
\hline Urinary retention & $1(2.1)$ & $1(2.1)$ & - & - & - \\
\hline Urinary urgency & $21(43.8)$ & $1(2.1)$ & - & - & - \\
\hline Ejaculation disorder $(n=20)$ & $5(25)$ & $1(5)$ & - & - & - \\
\hline Erectile disfunction ( $n=20$ ) & $2(10.0)$ & $6(30.0)$ & $2(10.0)$ & - & - \\
\hline Dyspareunia (n=18) & $1(5.6)$ & $2(11.1)$ & $1(5.6)$ & - & - \\
\hline Vaginal dryness $(n=18)$ & $1(5.6)$ & $3(16.7)$ & $1(5.6)$ & - & - \\
\hline Vaginal stricture $(n=18)$ & $1(5.6)$ & $1(5.6)$ & $1(5.6)$ & - & - \\
\hline Treatment-related secondary malignancy & - & - & $1(2.0)$ & - & - \\
\hline Other** & - & - & - & - & - \\
\hline
\end{tabular}

* = data not available for all patients; ${ }^{* *}=$ other: anal pain, anal, rectal or colonic haemorrhage, anal necrosis, anal or rectal fissure, anal ulcer, rectal obstruction or stenosis

CTCAE = Common Terminology Criteria for Adverse Events version 5.0.; G = grade

from 7.5 pre-treatment to 21.5 with $>5$-year follow up $(\mathrm{p}=0.008)$. T1 and T5 comparison showed a small but significant deterioration of pain, fatigue and nausea $(\mathrm{p}=0.09,0.017$ and 0.033 , respectively) after treatment with longer follow-up.

According to QLQ-C30, our patient cohort had significantly lower QoL in comparison to the general Slovenian population (Table 4). Nearly all functional scales' mean scores were lower with the exception of emotional function. Patients reported more fatigue, constipation, diarrhoea and financial problems. Compared to EORTC reference values for colorectal (CRC) cancer patients, our cohort had borderline significant lower cognitive functioning and reported higher financial problems. 
TABLE 4. Health-related quality of life analysis: Mean scores comparisons 5 years after treatment with general Slovenian population ${ }^{38}$ and with EORTC reference values for colorectal cancer patients 39 for all scales of EORTC QLQ-C30

\begin{tabular}{|c|c|c|c|c|c|}
\hline Scale & $\begin{array}{l}5 \text {-year post-surgery } \\
\text { mean (SD) }\end{array}$ & $\begin{array}{c}\text { General Slovenian } \\
\text { population mean (SD) }\end{array}$ & p value* & $\begin{array}{l}\text { Colorectal reference } \\
\text { values mean (SD) }\end{array}$ & p value* \\
\hline Global health status/QoL & $60.8(26.1)$ & $71.1(21.4)$ & 0.035 & $62.1(23.4)$ & 0.759 \\
\hline Physical function & $78.9(24.5)$ & $91.8(14.0)$ & 0.006 & $83.0(21.1)$ & 0.285 \\
\hline Role function & $77.4(26.0)$ & $88.7(20.1)$ & 0.022 & $70.4(32.8)$ & 0.238 \\
\hline Cognitive function & $78.0(24.5)$ & $90.2(16.0)$ & 0.009 & $85.2(20.4)$ & 0.052 \\
\hline Social function & $78.5(24.8)$ & $90.9(17.3)$ & 0.009 & $76.0(28.6)$ & 0.629 \\
\hline \multicolumn{6}{|l|}{ Symptom scales } \\
\hline Fatigue & $29.4(23.2)$ & $19.8(19.8)$ & 0.029 & $34.7(28.4)$ & 0.302 \\
\hline Nausea/vomiting & $6.5(10.3)$ & $3.3(10.6)$ & 0.097 & $7.3(17.2)$ & 0.796 \\
\hline Appetite loss & $12.9(22.2)$ & $5.3(15.5)$ & 0.067 & $19.1(30.2)$ & 0.256 \\
\hline Constipation & $20.4(26.8)$ & $6.9(16.9)$ & 0.009 & $15.8(27.9)$ & 0.363 \\
\hline Diarrhoea & $16.1(22.6)$ & $4.2(13.6)$ & 0.006 & $16.6(27.6)$ & 0.920 \\
\hline Financial problems & $22.6(29.0)$ & $6.6(17.5)$ & 0.005 & $13.6(26.3)$ & 0.059 \\
\hline
\end{tabular}

$*$ values $(p<0.050)$ are bolded

\section{Discussion}

Preoperative use of IMRT for LARC is increasing rapidly with great variations in clinical practice among centres ${ }^{24}$ indicating a lack of quality clinical studies reporting treatment outcome and toxicity for different fractionation regimes. To date, only four prospective phase II studies have been published with short-term outcome data after preoperative IMRT concomitant with capecitabine in patients with LARC $9,16,25,26$, but none of them reported long-term results. Our study is the first to report a 5-year treatment outcome with late toxicity and QoL.

With shorter treatment time and no dose escalation with SIB to primary tumour only, our pCR rate improved from $10 \%$ to $25.5 \% .{ }^{16,27}$ With dose escalation in a Chinese study (41 Gy elective; 56 Gy tumour/lymph node; 22 fractions) and a Spanish study (46 Gy elective; 57.5 Gy tumour/lymph nodes; 23 fraction) they reported $31 \%$ and $30.6 \%$ pCR, respectively.9,11 The higher pCR rate can reflect higher BED in these trials, but pCR of $32.6 \%$ recently reported by Simson et. al. in a prospec- tive observational study with single target dose of 50.4 Gy in 28 fractions suggests other possible factors influencing treatment results, since there are important differences in target definition and treatment verification between studies. ${ }^{28}$ With no boost to pathologic lymph nodes, but with detailed contouring guidelines, added internal safety margin and image-guided radiation therapy (IGRT), we have achieved an equal or better $\mathrm{N}$ downstaging rate $(83 \%)$ in comparison to the Chinese and Spanish trials $(79.2 \%$ and $47.2 \%$, respectively) where a $5 \mathrm{~mm}$ uniform margin was used.

The encouraging total downstaging rate of $87 \%$ in our study translated into excellent 5-year OS, DFS and LC of $80.9 \%, 77.1 \%$ and $95.2 \%$, respectively. OS and DFS were significantly higher compared to our historic cohort $^{29}$ (OS 61.4\%, p $=0.03$ and DFS $52.4 \%, p=0.01)$. Studies with more intensified escalated IMRT regimes with added concurrent oxaliplatin ${ }^{12,30}$, are reporting 5-year OS of $63-82 \%$ and 5 -year DFS of $60-66 \%$, that are comparable to our study.

A reported death from cardiovascular disease in three male patients can probably explain why 
male gender is significant prognostic factor for OS but not for DFS. Surprisingly, the type of operation significantly affected OS and DFS. In concordance with other studies, we found significant association between total downstaging and $\mathrm{pN} 0$ with the improvement of $\mathrm{DFS}^{31}$, but we found no predictive value for $\mathrm{pCR}$, TRG prognostic group ${ }^{19}$ or NAR prognostic group ${ }^{32}$ as survival surrogates. However, we found excellent prognosis for the group of patients with pCR who had 91.7\% 5-year OS and 100\% 5-year DFS and 100\% 5-year LC, confirming observations from other studies. ${ }^{33}$

Previously, we reported low acute toxicity of preoperative treatment and postoperative complications ( $\mathrm{G} \geq 3$ of $4 \%$ and $8 \%$ respectively). All patients with LAR had protective stoma placement, so we recorded no anastomotic leakage, but with time, $4(8 \%)$ cases of anastomotic dehiscence were detected, as expected from the published literature. ${ }^{34}$ In median follow-up of 70 months, we recorded $17 \mathrm{G} \geq 3$ late adverse events in 12 patients (25\% of patients who underwent surgery), with $15 \%$ of GI, $2 \%$ urinary and $8 \%$ sexual late toxicity, significantly lower than to our historic cohort $(40 \%$, $19.2 \%$ and $51.7 \%$, respectively $)^{29}$ and less than $35 \%$ reported late surgical complications after 3D CRT concomitant with 5-Fu/oxaliplatin. ${ }^{35}$ The only comparable IMRT study reporting late adverse events is a Belgian study, with preoperative IMRT-SIB to 46/55.2 Gy in 23 fractions and median 54 months of follow-up. ${ }^{10}$ Their estimated $G \geq 3$ late toxicity was $13 \%$ and is lower than the $25 \%$ observed in our study with much lower GI and GU toxicity rates ( $9 \%$ vs. $15 \%$ and $4 \%$ vs. $10 \%$, respectively) compared to ours. With different recording of late effects in our study, the late toxicity could have been overestimated. Anastomotic dehiscence was discovered late, when protective stoma closure was planned and was not counted as a postoperative complication. Late events were recorded with the actuarial method, so faecal incontinence, although not present at the time of the last follow-up, occurred previously in two patients. Also, we had no data on sexual activity and GI disorder prior to treatment, so all GI events are counted as late sequelae, although no difference in sexual functioning before and 5 years after treatment was found on QoL analysis. Exclusion of anastomotic dehiscence and two cases of faecal incontinence decreases our rate of GI G $\geq 3$ toxicity to $8 \%$, which is in concordance with $9 \%$ in the Belgian study and $9 \%$ reported after 3D CRT. ${ }^{34}$ The occurrence of $\mathrm{G} \geq 2$ late diarrhoea $(10 \%)$ is also within the range of reported rates in the literature, with $9.5 \%$ of patients from the EORTC 22921 trial who reported grade 2 diarrhoea or higher after 5-year follow-up. ${ }^{34}$ The Spanish and Chinese investigators reported 2 and 4 fistulas in median follow-up at $17 \mathrm{~m}$ and $22 \mathrm{~m}$. The occurrence of fistula in our study is similar, with 3 fistulas that occurred 29, 34 and 36 months after surgery, showing the importance of longer followup and subsequently underreporting of long toxicity events in clinical studies. Since the first publication with a reported sphincter preservation rate of $62 \%$ in our series, 6 patients with LAR ended up with permanent stoma due to faecal incontinence, anastomotic dehiscence and fistula, but still, we report a high rate of $5 \mathrm{y}$ colostomy-free survival of $76 \%$, comparable to other studies. ${ }^{35}$ Regarding major toxicity, 1 patient ( $2 \%$ ) died due to treatment-related toxicity, consistent with a $1.4 \%$ and $2 \%$ death rate in the EORTC 22921 and German CAO/ARO/ AIO-94 trial, respectively..$^{34,36}$

Our patient cohort have significantly lower quality of life compared to the general Slovenian population ${ }^{37}$ according to EORTC QLQ-C30 scores comparison, with inferior global function and functional mean scales with problems with fatigue, constipation, diarrhoea and finance. However, comparison to EORTC reference scores ${ }^{38}$ for CRC patients shows no difference in any of the QLQ-C30 items with borderline lower cognitive function, reflecting advanced patient age (median 75 years) at 5-year data collection. Time analysis of EORTC QLQ-C30 and QLQ-C29 median scores showed that improvement or deterioration of function/ symptom appeared one year after treatment and remained stable with longer follow-up. We recorded improvement in body image and a drop of anxiety and as expected after surgery, there was less mucus/blood in stool, and after radiation patients reported hair loss. A significant rise in reported faecal incontinence/leakage, is in concordance with the reported late toxicity but late detection of urinary incontinence (significant after 5 but not 1 year) indicates the importance of long follow-up for reliable reporting of late toxicity. Contrary to $8 \%$ sexual late toxicity findings, there were no differences in sexual end points in the QoL analysis, reflecting the possibility of overestimation of these late events in our cohort.

Together with uncertainty in the reporting of late toxicity, the main limitation of our study design is the small sample size and lack of randomization. According to definition of "locally advanced rectal cancers" before new subgroup division consensus in 2013, we used the term locally advanced also for the intermediate/bad group, without additional 
subdivision of T3 tumours. Nevertheless, our results are comparable to above mentioned studies, for they used the same definition in that time. The advantages of our study are the very strict radiotherapy protocol and quality control with the precise recording of acute and late toxicity events. In comparison to other IMRT studies for preoperative LARC, we were the only one not to intensify treatment with dose escalation and/or novel drug addition.

By shortening the overall treatment time using SIB, we reported excellent $25.5 \%$ pCR and after 5-year follow-up, our OS and DFS $(80.9 \%$ and $77.1 \%$, respectively) are in the survival range of more intensified treatments ${ }^{12,30}$, suggesting possible overtreatment for certain patients with LARC. In the era of high local control, more effort should be directed to reducing acute and late toxicity. Our fractionation regime showed a very low acute toxicity profile with a non-negligible late events rate. More high-quality data with longer follow-up is needed to determine the true effect on QoL and possibly determine relevant tolerances of the organ at risk for late consequences to optimize treatment planning.

\section{Conclusions}

The results of this long-term study confirm that IMRT SIB is feasible for preoperative treatment of intermediate/locally advanced rectal cancer. By shortening the overall treatment time, without dose escalation, we achieved high $\mathrm{pCR}$, five-year overall survival, disease-free survival and local control. Due to the favourable acute toxicity profile, our treatment regime is suitable for treatment intensification with another drug in addition to capecitabine. More long-term data is needed for late toxicity assessment.

\section{References}

1. Gérard JP, Azria D, Gourgou-Bourgade S, Martel-Lafay I, Hennequin C, Etienne PL, et al. Clinical outcome of the ACCORD 12/0405 PRODIGE 2 randomized trial in rectal cancer. J Clin Oncol 2012; 30: 4558-65. doi: 10.1200/ JCO.2012.42.8771

2. Rödel C, Liersch T, Becker H, Fietkau R, Hohenberger W, Hothorn T, et al. Preoperative chemoradiotherapy and postoperative chemotherapy with fluorouracil and oxaliplatin versus fluorouracil alone in locally advanced rectal cancer: initial results of the German CAO/ARO/AIO-04 randomised phase 3 trial. Lancet Oncol 2012; 13: 679-87. doi: 10.1016/S1470-2045(12)70187-0

3. O'Connell MJ, Colangelo LH, Beart RW, Petrelli NJ, Allegra CJ, Sharif S, et al. Capecitabine and oxaliplatin in the preoperative multimodality treatment of rectal cancer: surgical end points from National Surgical Adjuvant Breast and Bowel Project trial R-04. J Clin Oncol 2014; 32: 1927-34. doi: 10.1200/ JCO.2013.53.7753
4. Bahadoer RR, Dijkstra EA, van Etten B, Marijnen CAM, Putter $H$, Kranenbarg $E M$, et al. Short-course radiotherapy followed by chemotherapy before total mesorectal excision (TME) versus preoperative chemoradiotherapy, TME, and optional adjuvant chemotherapy in locally advanced rectal cancer (RAPIDO): a randomised, open-label, phase 3 trial. Lancet Oncol 2021; 22: 29-42. doi: 10.1016/S1470-2045(20)30555-6

5. Arbea L, Ramos LI, Martínez-Monge R, Moreno M, Aristu J. Intensitymodulated radiation therapy (IMRT) vs. 3D conformal radiotherapy (3DCRT) in locally advanced rectal cancer (LARC): dosimetric comparison and clinical implications. Radiat Oncol 2010; 5: 17. doi: 10.1186/1748-717X-5-17

6. Guerrero Urbano MT, Henrys AJ, Adams EJ, Norman AR, Bedford JL, Harrington $\mathrm{KJ}$, et al. Intensity-modulated radiotherapy in patients with locally advanced rectal cancer reduces volume of bowel treated to high dose levels. Int J Radiat Oncol Biol Phys 2006; 65: 907-16. doi: 10.1016/j. ijrobp.2005.12.056

7. Engels B, De Ridder M, Tournel K, Sermeus A, De Coninck P, Verellen D, et al. Preoperative helical tomotherapy and megavoltage computed tomography for rectal cancer: impact on the irradiated volume of small bowel. Int $J$ Radiat Oncol Biol Phys 2009; 74: 1476-80. doi: 10.1016/j.jjrobp.2008.10.017

8. Mok $\mathrm{H}$, Crane $\mathrm{CH}$, Palmer MB, Briere TM, Beddar S, Delclos ME, et al. Intensity modulated radiation therapy (IMRT): differences in target volumes and improvement in clinically relevant doses to small bowel in rectal carcinoma. Radiat Oncol 2011; 6: 63. doi: 10.1186/1748-717X-6-63

9. Li JL, Ji JF, Cai Y, Li XF, Li YH, Wu H, et al. Preoperative concomitant boost intensity-modulated radiotherapy with oral capecitabine in locally advanced mid-low rectal cancer: a phase II trial. Radiother Oncol 2012; 102: 4-9. doi: 10.1016/j.radonc.2011.07.030

10. Engels B, Platteaux N, Van den Begin R, Gevaert T, Sermeus A, Storme G, et al. Preoperative intensity-modulated and image-guided radiotherapy with a simultaneous integrated boost in locally advanced rectal cancer: report on late toxicity and outcome. Radiother Oncol 2014; 110: 155-9. doi: 10.1016/j. radonc.2013.10.026

11. Hernando-Requejo O, López M, Cubillo A, Rodriguez A, Ciervide R, Valero $\mathrm{J}$, et al. Complete pathological responses in locally advanced rectal cancer after preoperative IMRT and integrated-boost chemoradiation. Strahlenther Onkol 2014; 190: 515-20. doi: 10.1007/s00066-014-0650-0

12. Picardi V, Macchia G, Guido A, Giaccherini L, Deodato F, Farioli A, et al. Preoperative chemoradiation with VMAT-SIB in rectal cancer: a phase II study. Clin Colorectal Cancer 2017; 16: 16-22. doi: 10.1016/j. clcc.2016.06.004

13. Hong YS, Nam BH, Kim KP, Kim JE, Park SJ, Park YS, et al. Oxaliplatin, fluorouracil, and leucovorin versus fluorouracil and leucovorin as adjuvant chemotherapy for locally advanced rectal cancer after preoperative chemoradiotherapy (ADORE): an open-label, multicentre, phase 2, randomised controlled trial. Lancet Oncol 2014; 15: 1245-53. doi: 10.1016/S14702045(14)70377-8

14. Zhu J, Liu F, Gu W, Lian P, Sheng W, Xu J, et al. Concomitant boost IMRT-based neoadjuvant chemoradiotherapy for clinical stage II/III rectal adenocarcinoma: results of a phase II study. Radiat Oncol 2014; 9: 70. doi: 10.1186/1748717X-9-70

15. Hong TS, Moughan J, Garofalo MC, Bendell J, Berger AC, Oldenburg NB, et al. NRG Oncology Radiation Therapy Oncology Group 0822: a phase 2 study of preoperative chemoradiation therapy using intensity modulated radiation therapy in combination with capecitabine and oxaliplatin for patients with locally advanced rectal cancer. Int J Radiat Oncol Biol Phys 2015; 93: 29-36. doi: 10.1016/j.ijrobp.2015.05.005

16. But-Hadzic J, Anderluh F, Brecelj E, Edhemovic I, Secerov-Ermenc A, Hudej $R$, et al. Acute toxicity and tumor response in locally advanced rectal cancer after preoperative chemoradiation therapy with shortening of the overall treatment time using intensity-modulated radiation therapy with simultaneous integrated boost: a phase 2 trial. Int J Radiat Oncol 2016; 96: 1003-10. doi: 10.1016/j.jirobp.2016.08.031

17. But-Hadzic J, Velenik V. Preoperative intensity-modulated chemoradiation therapy with simultaneous integrated boost in rectal cancer: 2-year followup results of phase II study. Radiol Oncol 2018; 52: 23-29. doi: 10.1515/ raon-2018-0007

18. Edge SB, Compton CC. The American Joint Committee on Cancer: the 7th edition of the AJCC cancer staging manual and the future of TNM. Ann Surg Oncol 2010; 17: 1471-4. doi: 10.1245/s10434-010-0985-4 
19. Dworak O, Keilholz L, Hoffmann A. Pathological features of rectal cancer after preoperative radiochemotherapy. Int J Colorectal Dis 1997; 12: 19-23. doi: $10.1007 / \mathrm{s} 003840050072$

20. Cancer Institute N. Common Terminology Criteria for Adverse Events (CTCAE) v5.0.; 2017. [cited 2021 Feb 04]. Available at https://ctep.cance. gov/protocoldevelopment/electronic applications/docs/ctcae v5 quick reference_5x7.pdf

21. Aaronson NK, Ahmedzai S, Bergman B, Bullinger M, Cull A, Duez NJ, et al. The European Organization for Research and Treatment of Cancer QLQ-C30: a quality-of-life instrument for use in international clinical trials in oncology. J Natl Cancer Inst 1993; 85: 365-76. doi: 10.1093/jnci/85.5.365

22. van der Hout A, Neijenhuijs KI, Jansen F, van Uden-Kraan CF, Aaronson NK, Groenvold $\mathrm{M}$, et al. Measuring health-related quality of life in colorectal cancer patients: systematic review of measurement properties of the EORTC QLQ-CR29. Support Care Cancer 2019; 27: 2395-412. doi: 10.1007/ s00520-019-04764-7

23. Fayers $\mathrm{P}$, Aaronson NK, Bjordal $\mathrm{K}$, Groenvold $\mathrm{M}$, Curran $\mathrm{D}$, Bottomley $\mathrm{A}$. EORTC QLQ-C3O Scoring Manual. European Organisation for Research and Treatment of Cancer; 2001. [cited 2021 Feb 04]. Available at https:// abdn.pure.elsevier.com/en/publications/eortc-qlq-c30-scoring-manual-3rdedition.

24. Hanna CR, Slevin F, Appelt A, Beavon M, Adams R, Arthur C, et al. Intensitymodulated radiotherapy for rectal cancer in the UK in 2020. Clin Oncol 2021; 33: 214-23. doi: 10.1016/j.clon.2020.12.011

25. Fernández-Martos C, Pericay C, Aparicio J, Salud A, Safont M, Massuti B, et al. Phase II, randomized study of concomitant chemoradiotherapy followed by surgery and adjuvant capecitabine plus oxaliplatin (CAPOX) compared with induction CAPOX followed by concomitant chemoradiotherapy and surgery in magnetic resonance imaging-defined, locally advanced rectal cancer: Grupo cancer de recto 3 study. J Clin Oncol 2010; 28: 859-65. doi: $10.1200 / J C O .2009 .25 .8541$

26. Tey J, Leong CN, Cheong WK, Sze TG, Yong WP, Tham IWK, et al. A phase trial of preoperative concurrent chemotherapy and dose escalated intensity modulated radiotherapy (IMRT) for locally advanced rectal cancer. J Cancer 2017; 8: 3114-21. doi: 10.7150/jca.21237

27. Velenik V, Anderluh F, Oblak I, Strojan P, Zakotnik B. Capecitabine as a radiosensitizing agent in neoadjuvant treatment of locally advanced resectable rectal cancer: prospective phase II trial. Croat Med J 2006; 47: 693-700.

28. Simson DK, Mitra $S$, Ahlawat $P$, Saxena U, Sharma MK, Rawat $S$, et al. Prospective study of neoadjuvant chemoradiotherapy using intensitymodulated radiotherapy and 5 fluorouracil for locally advanced rectal cancer - Toxicities and response assessment. Cancer Manag Res 2018; 10: 519-26. doi: 10.2147/CMAR.S142076

29. Velenik V, Oblak I, Anderluh F. Long-term results from a randomized phase II trial of neoadjuvant combined-modality therapy for locally advanced rectal cancer. Radiat Oncol 2010; 5: 88. doi: 10.1186/1748-717X-5-88

30. Garofalo M, Moughan J, Hong T, J.Bendell, A.Berger, F.Lerma, et al. RTOG 0822: a phase II study of preoperative (PREOP) chemoradiotherapy (CRT) utilizing IMRT in combination with capecitabine $(C)$ and oxaliplatin $(O)$ for patients with locally advanced rectal cancer. Int J Radiat Oncol 2011; 81: S3-S4 doi: 10.1016/j.ijrobp.2011.06.008

31. Fokas E, Liersch T, Fietkau R, Hohenberger W, Beissbarth T, Hess C, et al. Tumor regression grading after preoperative chemoradiotherapy for locally advanced rectal carcinoma revisited: updated results of the CAO/ARO/AIO94 trial. J Clin Oncol 2014; 32: 1554-62. doi: 10.1200/JCO.2013.54.3769

32. George TJ, Allegra CJ, Yothers G. Neoadjuvant Rectal (NAR) Score: a new surrogate endpoint in rectal cancer clinical trials. Curr Colorectal Cancer Rep 2015; 11: 275-80 doi: 10.1007/s11888-015-0285-2

33. Maas M, Nelemans PJ, Valentini V, Das P, Rödel C, Kuo L, et al. Long-term outcome in patients with a pathological complete response after chemoradiation for rectal cancer: a pooled analysis of individual patient data. Lancet Oncol 2010; 11: 835-44. doi: 10.1016/S1470-2045(10)70172-8

34. Sauer R, Becker H, Hohenberger W, Rödel C, Wittekind C, Fietkau R, et al. Preoperative versus postoperative chemoradiotherapy for rectal cancer. $N$ Engl J Med 2004; 351: 1731-40. doi: 10.1056/NEJMoa040694

35. Urso E, Serpentini S, Pucciarelli $S$, De Salvo GL, Friso ML, Fabris G, et al. Complications, functional outcome and quality of life after intensive preoperative chemoradiotherapy for rectal cancer. Eur J Surg Oncol 2006; 32 1201-8. doi: 10.1016/j.ejso.2006.07.003
36. Bosset JF, Collette L, Calais G, Mineur L, Maingon P, Radosevic-Jelic L, et al. Chemotherapy with preoperative radiotherapy in rectal cancer. $N$ Engl $J$ Med 2006; 355: 1114-23. doi: 10.1056/NEJMoa060829

37. Velenik V, Secerov-Ermenc A, But-Hadzic J, Zadnik V. Health-related quality of life assessed by the EORTC QLQ-C30 questionnaire in the general slovenian population. Radiol Oncol 2017; 51: 342-50. doi: 10.1515/ raon-2017-0021

38. Scott NW, Fayers PM, Aaronson NK, Bottomley A, de Graeff A, Groenvold $\mathrm{M}$, et al; EORTC Quality of Life Group. EORTC QLQ-C3O Reference Values; 2008. [cited $2021 \mathrm{Feb}$ 04]. Available at https://www.eortc.org/app/uploads/ sites/2/2018/02/reference_values_manual2008.pdf 